\title{
Fístula portobiliar como complicación mayor de biopsia hepática transpercutánea bajo marcación ecográfica
}

\author{
Francisca Rivera O. ${ }^{1}$ y Diego San Martín R. ${ }^{2}$
}

\section{Arteriobiliary fistula as a several complication of ultrasonography guided liver percutaneous biopsy}

Woman of 71 years, in study of chronic liver disease, Child-Pugh A, computed tomography (CT) suggestive of cirrhosis, autoimmune hepatitis score probable. It was decided to perform a liver percutaneous biopsy for diagnosis and treatment. After the procedure, the patient presented a self-limited episode of hematemesis. Upper digestive endoscopy without blood or other injury, abdominal ultrasound without free liquid. The patient was hospitalized, evolving hemodynamically stable without new bleeding episodes. Medical discharge was decided after 24 hours of observation. The patient consults the next day at emergency room because of intense abdominal pain and anemia. CT Impressed a dilated bile duct with hyperdense content inside, so she was rehospitalized. The patient evolved with melaena and hypotension. Biliary hematoma due to arteriobiliary fistula was suspected. CT angiogram evidenced portobiliary fistula. It was managed with endovascular therapy but the patient remained hypotense with a requirement for vasoactive drugs and multi-organ dysfunction, and she finally died.

Key words: Liver biopsy, major complications, arteriobiliar fistula.

\section{Resumen}

Mujer de 71 años en estudio de enfermedad hepática crónica Child-Pugh A, tomografía computada (TC) sugerente de cirrosis, con puntaje probable de hepatitis autoinmune en su estudio etiológico. Se decidió realizar una biopsia hepática por punción para diagnóstico e iniciar tratamiento. Luego del procedimiento, presentó episodio autolimitado de hematemesis. Endoscopia digestiva alta sin contenido de sangre ni otras lesiones, ecotomografía abdominal sin líquido libre. Se hospitaliza, evolucionando hemodinámicamente estable sin nuevos episodios de hematemesis. Se decide alta tras 24 h de observación. Consultó en urgencias un día después por dolor epigástrico intenso y anemia. TC abdomen y pelvis impresionó dilatación de vía biliar con contenido hiperdenso en su interior por lo que se rehospitaliza. Evolucionó con melena e hipotensión. Se sospecha hematoma biliar por fístula arteriobiliar. AngioTC computarizada de abdomen y pelvis, evidenció fístula portobiliar. Se maneja con terapia endovascular, pero paciente grave, con requerimiento de drogas vasoactivas y disfunción multiorgánica por lo que finalmente fallece.

Palabras clave: Biopsia hepática, complicaciones mayores, fistula arteriobiliar.

\section{Introducción}

La biopsia hepática percutánea es una herramienta relevante en el estudio, manejo y seguimiento de la enfermedad hepática crónica (EHC). Aunque su implementación data desde 1883, esta técnica sigue estando ampliamente vigente y aceptada en el mundo. Se considera un procedimiento seguro con una mortalidad estimada menor a 1/10.000 pacientes ${ }^{1}$. Las complicaciones menores ocurren en $30 \%$ de los pacientes y son: dolor, malestar general e hipotensión por reflejo vasovagal ${ }^{2,3}$. Las complicaciones mayores ocurren en $0,2-0,8 \%$ de los pacientes destacando la hemorragia como la más relevante por ser la principal causa de mortalidad ${ }^{2,3}$. Esta se manifiesta con sangrado grave que requiere soporte transfusional y otras intervenciones en $1 / 2.500$ a $1 / 10.000$ pacientes 1 . Además, se ha descrito que $61 \%$ de las complicaciones ocurren en las primeras $2 \mathrm{~h}$ después del procedimiento lo que justifica protocolos ambulatorios ${ }^{4}$. A continuación, se presenta un caso de fistula portobiliar secundario a biopsia hepática percutánea bajo marcación ecográfica.
IInterna de Medicina Universidad de Concepción. Concepción, Chile. ${ }^{2}$ Gastroenterólogo Hospital Las Higueras. Talcahuano, Chile.

Recibido: 30 de junio de 2020

Aceptado: 1 de noviembre de 2020

\section{Correspondencia a:} Dr. Diego San Martín Rodríguez Unidad de

Gastroenterología Hospital Las Higueras de Talcahuano. Talcahuano, Chile. dlsanmartin@uc.cl 


\section{Caso Clínico}

\section{Caso clínico}

Paciente sexo femenino de 71 años con antecedente de hipotiroidismo, colecistectomizada con vía biliar intra y extrahepática de calibre normal, en estudio de EHC por elevación persistente de aminotranferasas. Tomografía computada (TC) sugerente de cirrosis, sin ascitis ni hepatocarcinoma. Endoscopia digestiva alta sin várices esofágicas. Exámenes de laboratorio destacaba INR 1,3, Hb 12,1, HTO 33,9, Plaquetas 77.000, albumina 3,7, GOT 41, GPT 26,4, GGT 130, FA 87, Bilirrubina total 1,98, bilirrubina directa 0,77. Child Pugh A, Meld-Na 11. Sin antecedente de sangrado digestivo ni uso de aspirina ${ }^{\circledR}$ o anticoagulantes. Estudio inmunológico destacó ANA 1/320, IGG > 1,1 veces, AMA (-), ASMA (-), ANCA 1/320, virus hepatitis no reactivos. Por score abreviado de hepatitis autoinmune sugerente, se decidió realizar biopsia hepática percutánea para confirmar diagnóstico y poder iniciar terapia inmunomoduladora. Se lleva a cabo procedimiento bajo marcación ecográfica con técnica de Menghini, en dos pases, sin incidentes obteniéndose una muestra escasa.
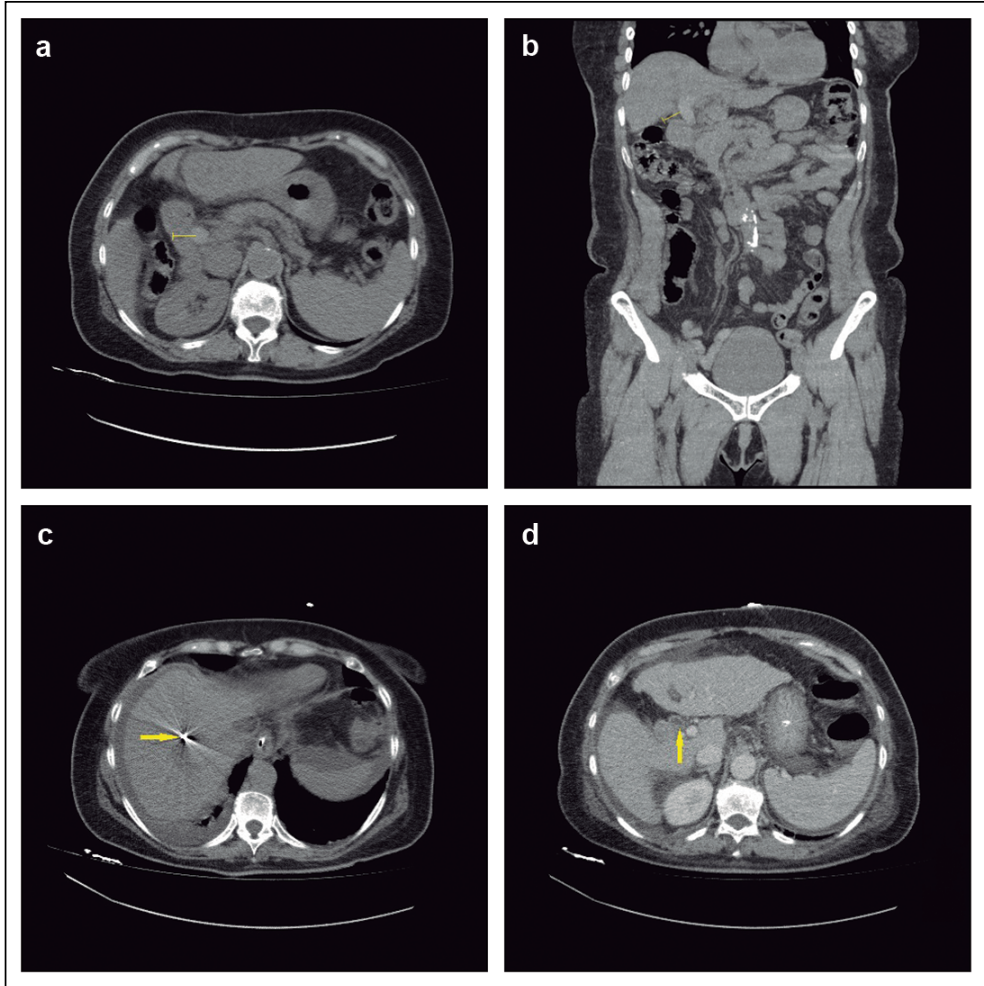

Figura 1. Tomografía computarizada de abdomen, fase sin contraste corte axial (a) y coronal (b). Flecha indica vía biliar ocupada por sangre; (c) Tomografía computarizada de abdomen luego de tratamiento endovascular, fase sin contraste. Flecha indica coil in situ; (d) Tomografía computarizada de abdomen control post procedimiento, fase sin contraste. Flecha indica vía biliar sin contenido hemático.
Durante la primera hora, la paciente evoluciona con episodio de hematemesis autolimitada sin melena en tacto rectal, asociado a hipotensión (PA 70/40 $\mathrm{mmHg}$ ) con buena perfusión distal (llene capilar 2 segundos) que respondió favorablemente a volemización. Dado que paciente aún estaba bajo sedación anestésica, se decidió realizar endoscopia digestiva alta inmediata que resultó sin contenido de sangre ni otras lesiones. Ecografía abdominal no reveló líquido libre perihepático. Monitorización hospitalizada con evolución hemodinámicamente estable, con leve dolor en el sitio de punción sin nuevos eventos de hematemesis, dando el alta tras $24 \mathrm{~h}$ de observación con $\mathrm{Hb}$ 10,8, HTO 30,1.

Paciente consultó en servicio de urgencia un día después por cuadro de dolor abdominal intenso en epigastrio asociado a anemia ( $\mathrm{Hb} \mathrm{7,6} \mathrm{mg/dL).} \mathrm{Al}$ examen físico destacó abdomen blando, doloroso a la palpación. Exámenes de laboratorio: bilirrubina total de 6,54 , bilirrubina directa de 4,89, fosfatasa alcalina de 97, GGT 226, GTP 105, GOT 166, plaquetas 72.000 y parámetros inflamatorios bajos. Se realizó TC abdomen que impresionó dilatación de la vía biliar con contenido hiperdenso en su interior por lo que se rehospitaliza.

Paciente evolucionó con deterioro del estado de conciencia y episodios de melena con tendencia a hipotensión. Evaluada por equipo de cirugía hepatobiliar que sugieren hematoma biliar con hemobilia por probable fístula arteriobiliar. AngioTC abdomen y pelvis (Figura 1), con fases arterial y venosa, evidenció fístula portobiliar pequeña desde porta derecha. Se decide terapia endovascular con embolización de lesión, instalándose coils sin incidentes. Pese a evolución sin sangrado activo ni nuevos requerimientos transfusionales, paciente persiste grave, hipotensa, con requerimiento de drogas vasoactivas y disfunción multiorgánica por lo que finalmente fallece.

\section{Discusión}

Las complicaciones mayores asociadas a la biopsia hepática son escasas. La hemobilia se produce cuando existe una fístula entre la circulación portal y el sistema biliar. Esta ocurre con una frecuencia del $0,059 \%$ de los casos, pudiendo ser una complicación tardía ${ }^{5}$. La clínica clásicamente descrita es la tríada de Quincke caracterizada por dolor abdominal superior, hemorragia digestiva alta e ictericia, aunque las tres están presentes de forma concomitante en sólo el $22 \%$ de los pacientes ${ }^{6}$. Además, pueden producirse como complicaciones a la hemobilia cuadros como colecistitis, colangitis y pancreatitis ${ }^{7}$.

La morbimortalidad depende del pronto manejo del sangrado, sin embargo, el diagnóstico no siempre 
es fácil debido a la baja sensibilidad de las pruebas diagnósticas8. Por lo anterior se requiere una alta sospecha clínica, sobre todo en paciente con antecedentes de procedimientos en vía hepática.

Hasta un $60 \%$ de los casos de hemobilia se pueden diagnosticar usando la endoscopia digestiva alta ${ }^{9}$. Se podría requerir uso de duodenoscopia para visualizar la papila mayor en búsqueda de coágulos u otra evidencia de sangrado. La colangiopancreatografía retrograda puede ser utilizada para visualizar el árbol biliar y también puede ofrecer opciones terapéuticas si se asocia a obstrucción biliar ${ }^{10}$.

La ecografía abdominal es una técnica útil para establecer el diagnóstico, al detectar la presencia de coágulos en la vesícula o vía biliar, o demostrando lesiones estructurales que justifiquen el sangrado ${ }^{10}$.

La tomografía computarizada con protocolo de angiografía se ha convertido en el último tiempo en la primera elección diagnóstica para hemobilia ya que es una técnica no invasiva, con baja tasa de radiación en comparación a la angiografía convencional y con resultados rápidos y certeros ${ }^{10}$.

Sin embargo, la angiografía se mantiene como el gold standar para diagnóstico y tratamiento de estos cuadros $^{10}$. La evaluación angiográfica clásicamente realiza el siguiente estudio vascular: primero se evalúa el tronco celiaco, si este no revela sangrado se avanza el catéter para evaluar las arterias hepáticas (izquierda y derecha). Si aún no se revela sangrado, se estudia la arteria mesentérica superior. La extravasación de contraste al árbol biliar, truncamiento arterial periférico, sección arterial, pseudoaneurisma y fistula arterioportobiliar son sugerentes de lesión.

El tratamiento definitivo consiste en eliminar la causa de sangrado y depende de la severidad del sangrado y la situación general del paciente. Algunas opciones son la resección hepática, ligado directo de los vasos sangrantes, colecistectomía, resección pancreática entre otras. En general, se recomienda la embolización arterial selectiva siempre que se pueda, reservándose la cirugía para los casos de fracaso o complicación de la embolización ${ }^{10,11}$.

La embolización arterial selectiva tiene una tasa de éxito entre $80-100 \%{ }^{12}$. Una vez identificado el sitio de sangrado por angiografía, se selecciona la arteria afectada ubicando un micro catéter en la zona blanco a trabajar. Luego, se procede a la embolización transarterial usando coils.

En el caso presentado, la paciente no logro sobrevivir pese al tratamiento con embolización. Es probable que esto sea producto de la demora en el diagnóstico asociado a las comorbilidades del paciente, especialmente su cirrosis y edad avanzada. Esto reafirma lo anteriormente expuesto, respecto a que la alta sospecha diagnóstica es el principal factor pronóstico en estos pacientes para recibir un tratamiento oportuno.

Existen alternativas a la biopsia hepática percutánea que se prefieren en pacientes con mayor riesgo de sangrado. La biopsia hepática transyugular consiste en la obtención de un fragmento de tejido hepático mediante un abordaje transvenoso sin punción de la cápsula de Glisson, disminuyendo el riesgo de hemorragia. Su indicación fundamental, es la presencia de alteración grave de la coagulación. Tiene una incidencia de complicaciones inferior al $5 \%{ }^{13}$.

Existen complicaciones derivadas de la punción cervical, fundamentalmente hematomas, disfonía, parestesias del miembro superior y ocasionalmente síndrome de Horner por lesión del simpático cervical. El paso de los distintos dispositivos puede motivar arritmias auriculares que son habitualmente autolimitadas. La complicación más grave es la perforación capsula hepática dado que implica el riesgo de hemoperitoneo que se produce en $0,5 \%$ de los $\operatorname{casos}^{14}$.

El uso de marcación ecográfica ha aumentado la seguridad de la biopsia hepática percutánea al disminuir la tasa de complicaciones menores como dolor y días de hospitalización, sin embargo, no ha logrado reducir el riesgo de sangrado ${ }^{15}$. Se han establecido algunos factores de riesgo que se asocian a mayor incidencia de sangrado como son la edad avanzada, perfil de coagulación e INR alterado, cirrosis, mayor número de pases, entre otros ${ }^{16}$. Respecto al número de pases realizado, estudios han demostrado que 3 o más pases aumenta el riesgo de complicaciones menores, principalmente dolor, pero no de complicaciones severas, y que los pacientes con 2 pases tienen complicaciones comparables con los que recibieron 1 pase $^{17,18}$. Sigue siendo controversial que tan fiable serían estos factores como predictores ${ }^{19}$.

\section{Referencias}

1.- Rockey DC, Caldwell SH, Goodman ZD, Nelson RC, Smith AD; American Association for the Study of Liver Diseases. Liver biopsy. Hepatology. 2009;49:1017-44

2.- Kitchin DR, Del Rio AM, Woods M,
Ludeman L, Hinshaw JL. Percutaneous liver biopsy and revised coagulation guidelines: a 9-year experience. Abdom Radiol (NY). 2018;43: 1494-501.

3.- McGill DB, Rakela J, Zinsmeister AR,
Ott BJ. A 21-year experience with major hemorrhage after percutaneous liver biopsy. Gastroenterology. 1990; 99:1396400

4.- Nodarse-Pérez PO, Pérez-Menéndez R, Heredia-Andrade ED, Noa-Pedroso 


\section{Caso Clínico}

G, Araluce-Cordoví R, FernándezSotolongo J. Seguridad de la reducción del tiempo de reposo posbiopsia hepática percutánea y por vía laparoscópica. Cir Cir. 2016;84:196-202.

5.- Piccinino F, Sagnelli E, Pasquale G, Giusti G. Complications following percutaneous liver biopsy: a multicentre retrospective study on 68,276 biopsies. J Hepatol 1986;2:165.

6.- Green MHA, Duell RM, Johnson CD, Jamieson NV. Haemobilia. Br J Surg 2001;88:773-86.

7.- Van Os EC, Petersen BT. Pancreatitis secondary to percutaneous liver biopsyassociated hemobilia. Am J Gastroenterol 1996; 91: 577-80.

8.- Green MHA, Duell RM, Johnson CD, Jamieson NV. Haemobilia. Br J Surg 2001; 88: 773-86.

9.- Feng W, Yue D, ZaiMing L, ZhaoYu L, XiangXuan Z, Wei L, et al. Iatrogenic hemobilia: Imaging features and management with transcatheter arterial embolization in 30 patients. Diagn Interv Radiol 2016; 22: 371-7.

10.- Berry R, Han JY, Kardashian AA,
LaRusso NF, Tabibian JH. Hemobilia: Etiology, diagnosis, and treatment. Liver Res. 2018:200-8.

11.- Dousset B, Sauvanet A, Bardou M, Legmann P, Vilgrain V, Belghiti J. Selective surgical indications for iatrogenic hemobilia. Surgery. 1997;121:37-41.

12.- Zhornitskiy A, Berry R, Han JY, Tabibian JH. Hemobilia: Historical overview, clinical update, and current practices. Liver Int. 2019;39:1378-88.

13.- Papatheodoridis GV, Patch D, Watkinson A,Tibballs J, Burroughs AK.Transjugular liver biopsy in the 1990s: a 2-year audit. Aliment Pharmacol Ther 1999; 13: 603-8.

14.- McAfee JH, Keeffe EB, Lee RG, Rosch J. Transjugular liver biopsy. Hepatology 1992;15: 726-32.

15.- Boyum JH, Atwell TD, Schmit GD, Poterucha JJ, Schleck CD, Harmsen WS, et al. Incidence and Risk Factors for Adverse Events Related to ImageGuided Liver Biopsy. Mayo Clin Proc. 2016;91:329-35.

16.- Midia M, Odedra D, Shuster A,
Midia R, Muir J. Predictors of bleeding complications following percutaneous image-guided liver biopsy: a scoping review. Diagn Interv Radiol. 2019;25:7180.

17.- Chi H, Hansen BE, Tang WY, Schouten JN, Sprengers D, Taimr P, et al. Multiple biopsy passes and the risk of complications of percutaneous liver biopsy. Eur J Gastroenterol Hepatol. 2017;29:36-41.

18.- Van der Poorten D, Kwok A, Lam T, Ridley L, Jones DB, Ngu MC, et al. Twenty-year audit of percutaneous liver biopsy in a major Australian Teaching Hospital. Intern Med J 2006; 36:692-9.

19.- Strobel D, Bernatik T, Blank W, Will U, Reichel A, Wüstner M, et al. Incidence of bleeding in 8172 percutaneous ultrasound-guided intraabdominal diagnostic and therapeutic interventions - results of the prospective multicenter DEGUM interventional ultrasound study (PIUS study). Ultraschall Med. 2015;36: 122-31. 